\title{
BIOLOGÍA Y COMPORTAMIENTO DE Amblyseius chungas Denmark \& Muma (ACARI: PHYTOSEIIDAE) COMO PREDADOR DE Panonychus citri (McGregor) (ACARI: TETRANYCHIDAE)
}

\section{BIOLOGY AND BEHAVIOR OF Amblyseius chungas Denmark \& Muma (ACARI: PHYTOSEIIDAE) AS A PREDATOR OF Panonychus citri (McGregor) (ACARI: TETRANYCHIDAE)}

\author{
Alberto Daniel Guanilo y Norberta Martínez ${ }^{2}$
}

\begin{abstract}
Resumen
Panonychus citri (McGregor) es una plaga importante en los cítricos de la costa central del Perú. Amblyseius chungas Denmark \& Muma fue el ácaro predador más frecuente y abundante asociado a $P$. citri durante unas búsquedas realizadas en esa región. Para evaluar a $A$. chungas como controlador potencial de $P$. citri en cítricos, se estudió la duración de los estadios inmaduros de $A$. chungas y analizó el comportamiento predador de las hembras adultas de $A$. chungas cuando éstas se alimentan de ninfas y adultos $P$. citri como presa. Los experimentos fueron realizados en el laboratorio $\left(20-25^{\circ} \mathrm{C}\right.$ y $\left.70-80 \% \mathrm{HR}\right)$. El tiempo de desarrollo del huevo hasta la emergencia del adulto fue de $6.60 \pm 0.57$ y $6.46 \pm 0.44$ días en hembras y machos, respectivamente. Las hembras adultas de $A$. chungas alimentadas a diferentes densidades de hembras adultas y/o ninfas de $P$. citri como presa presentaron una respuesta funcional de tipo II basada en la clasificación propuesta por Holling. La oviposición fue de 1 a 3 huevos por día.

Palabras clave: Predación, respuesta funcional, arañita roja de los cítricos, Tetranychidae, ácaro predador, Phytoseiidae, cítricos, Perú
\end{abstract}

\begin{abstract}
Panonychus citri (McGregor) is an important citrus pest in the central coast of Peru. Amblyseius chungas Denmark \& Muma was the most frequent and abundant predatory mite during some surveys for natural enemies against $P$. citri in that region. We studied the duration of $P$. citri by immature stages of $A$. chungas and analyzed its predatory behavior when female adults of $A$. chungas were fed with $P$. citri nymphs and adults. The experiments were carried out in the laboratory $\left(20-25^{\circ} \mathrm{C}\right.$ and $\left.70-80 \% \mathrm{RH}\right)$. Development time from egg to adult was $6.60 \pm 0.57$ days in females and $6.46 \pm 0.44$ days in males. Female adults of $A$. chungas fed with different densities of adults and/or nymphs of $P$. citri showed a functional response type II according with the classification proposed by Holling. Oviposition of $A$. chungas ranged from 1 to 3 eggs/day.

Key words: Predation, functional response, citrus red mite, Tetranychidae, predatory mite, Phytoseiidae, citrus, Peru
\end{abstract}

\section{Introducción.}

La “arañita roja de los cítricos” Panonychus citri (McGregor) (Acari: Tetranychidae) es una plaga diseminada por los valles de la costa peruana comprendidos desde Piura hasta Tacna (Morín, 1985). Sus altas infestaciones están influenciadas por factores climáticos, presencia de estrés hídrico, y por depósitos de polvo (ProCitrus, 2005). Este ácaro se caracteriza por extraer la savia de las hojas, ocasionando la aparición de un punteado amarillento en el haz de las mismas. Altas densidades de $P$. citri pueden conducir a desecación y caída de hojas. Los frutos tiernos muestran también el punteado, y si la población del ácaro es elevada, el punteado persiste, adquiriendo tonos plateados, deteriorando la calidad comercial del fruto.
El control de $P$. citri mediante el uso de acaricidas resulta costoso y contribuye al desarrollo de resistencia, reduce la fauna de artrópodos benéficos, y genera la dispersión del ácaro problema (Chávez, 2003). Actualmente se realizan esfuerzos en la búsqueda de métodos alternativos naturales que ayuden a reducir sus altas infestaciones. El ácaro predador Amblyseius chungas Denmark \& Muma (Acari: Phytoseiidae) fue la especie más frecuente asociada a $P$. citri en la costa central del Perú durante unas búsquedas realizadas en esa región (Guanilo \& Martínez, 2007). Amblyseius chungas ha sido encontrado únicamente en Perú, en los Departamentos de Cuzco, Ica, La Libertad, Lambayeque, Lima, y San Martín (Denmark \& Muma, 1989; Guanilo \& Martínez, 2007; Guanilo et al., 2008). A pesar de la 
abundancia de esta especie y gran dispersión en Perú, no se conoce hasta el momento informaciones referidas a sus parámetros biológicos, ni su potencial como controlador biológico de arañitas rojas.

El objetivo del presente trabajo es determinar la duración de los estadios inmaduros de A. chungas y analizar el comportamiento predador de las hembras adultas de $A$. chungas mediante experimentos de respuesta funcional cuando éstas se alimentan de ninfas y adultos $P$. citri. Solomon (1949) definió la respuesta funcional como la relación entre el número de presas consumidas por un predador y la densidad de la presa, en un área e intervalo de tiempo fijos. Este análisis permite evaluar la potencialidad de agentes de control biológico de plagas.

\section{Materiales y métodos.}

La colecta de los ácaros se hizo cortando ramas de híbridos de mandarinas de la variedad Kara (M. Owari $\mathrm{x}$ M. King) infestadas con $P$. citri y A. chungas del Fundo Ramos $\left(13^{\circ} 04^{\prime} \mathrm{S}, \quad 76^{\circ} 21^{\prime} \mathrm{W}\right)$, Cañete, Departamento de Lima (Perú) en febrero de 2004, las cuales fueron colocadas en envases plásticos. El material fue transportado al laboratorio de Entomología de la Universidad Nacional Mayor de San Marcos (UNMSM) (Lima, Perú) donde se procedió a separar los ácaros usando un microscopio estereoscópico, para establecer colonias de $P$. citri y A. chungas.

La crianza de $P$. citri fue realizada sobre hojas de naranjo (Citrus sinensis L.) (Figura 1) provenientes de plantones mantenidos en un invernadero instalado en la UNMSM totalmente libres de pesticida. Las hojas fueron colocadas en bandejas de plástico (35 x 25 x 5 cm.) fabricado por Duraplast ${ }^{\circledR}$ (Lima, Perú), sobre una capa de algodón humedecido, colocando la parte adaxial (haz) de las hojas hacia arriba. Las hojas usadas fueron medianamente maduras y turgentes, previamente lavadas con $0.0005 \%$ de hipoclorito de sodio (lejía). Se usó aproximadamente entre 8-12 hojas por bandeja. Cada hoja fue infestada con 3-5 $P$. citri hembras adultas.

La crianza de los ácaros predadores $A$. chungas fue realizada sobre hojas provenientes de los mismos plantones usados en la crianza de $P$. citri, alimentados con diferentes estadios de $P$. citri dentro de envases de plástico translúcido Duraplast ${ }^{\circledR}(15 \times 10 \times 3 \mathrm{~cm})$ con 20 individuos alimentados con aproximadamente 200 $P$. citri diariamente.

Para las pruebas explicadas a continuación, las unidades experimentales, consistieron en envases de plástico ( $5 \mathrm{~cm}$ de diám. x $4 \mathrm{~cm}$ de altura) (Duraplast ${ }^{\circledR}$ ), cerrados con cinta adhesiva para evitar la fuga de los individuos evaluados. Cada envase donde fueron puestos los individuos evaluados tenía un disco de hoja de naranjo fresca de $3 \mathrm{~cm}$ de diám. colocando el haz hacia arriba. Debajo de la hoja fue colocado un papel filtro humedecido. Los discos de hojas fueron dejados en la unidad experimental durante el tiempo que duró el experimento. Las pruebas fueron realizadas en condiciones ambientales de $20-25^{\circ} \mathrm{C}$ y $70-80 \%$ HR aproximadamente. Durante las evaluaciones, los cambios de estadios de desarrollo fueron detectados mediante la observación de las mudas. Para diferenciar el sexo de los adultos, se hizo su respectivo montaje en medio de Hoyer.

1. Pruebas de duración de los estadios de desarrollo de Amblyseius chungas.

Huevos de $A$. chungas fueron aislados en las unidades experimentales individualmente. Luego que eclosionaban las larvas, se les suministraron treinta $P$. citri (ninfas). Como parámetros biológicos analizados se estimó la duración de los estadios de desarrollo por sexo. Las observaciones se realizaron cada 24 horas, para tal fin se procedía abrir la unidad experimental y observar el estadio de desarrollo y el número de presas consumidas a través de un microscopio estereoscopio. Para diferenciar cada estadio de desarrollo, se procedió a buscar la exuvia dejada en la unidad experimental.

2. Predación y reproducción de Amblyseius chungas. Se separaron hembras adultas de $A$. chungas provenientes de la crianza luego de aproximadamente 4-8 días que habían pasado al estado adulto y se procedió a suministrarles un número determinado de $P$. citri por un periodo de $24 \mathrm{~h}$. Pasado ese periodo se contabilizó la cantidad de $P$. citri consumidos y la cantidad de huevos ovipositados. Se realizaron tres experimentos: (2.1) Se expusieron individualmente hembras adultas de $A$. chungas a diferentes densidades de ninfas de $P$. citri $(1,5,10,15,20$ y 25). Fueron realizadas aproximadamente 25 repeticiones por prueba. (2.2) Se expusieron individualmente hembras adultas de $A$. chungas a diferentes densidades de hembras adultas de $P$. citri $(1,5,10,15,20,25,30$ y 50). Fueron realizadas aproximadamente 25 repeticiones por prueba. (2.3) Se expusieron diferentes densidades de $A$. chungas (1, 5 y 10 hembras adultas) a 50 hembras adultas de $P$. citri. Fueron realizadas de 14-26 repeticiones por prueba. Los experimentos (2.1) y (2.2) correspondieron a pruebas de la respuesta funcional.

Análisis de datos: todos los datos de los experimentos se procesaron estadísticamente, mediante un análisis de varianza y la prueba de Tukey, usando SPSS 17.0 (Creado por SPSS Inc.; Chicago, USA) como programa estadístico. Para evaluar la respuesta funcional, se procedió a ajustar una regresión logística, que permitiera determinar la forma de la curva de acuerdo a Juliano (1993). Luego, se estimaron los parámetros para la ecuación del tipo II mediante una regresión no lineal ajustada por cuadrados mínimos, usando como función general:

$$
N c=\mathrm{N}\{1-\exp [\mathrm{a}(\mathrm{TmNc}-\mathrm{T})]\},
$$

donde $N \boldsymbol{c}$ es el número de presas consumidas, $\boldsymbol{a}$ es la tasa de ataque constante (tasa de búsqueda 
instantánea), $\boldsymbol{N}$ es la densidad de la presa, $\boldsymbol{T}$ es el tiempo disponible y $\mathbf{T m}$ es el tiempo de manipulación.

Adicionalmente a los datos obtenidos durante los experimentos, se realizaron observaciones directas del comportamiento de $A$. chungas en las colonias mantenidas en el laboratorio y con material traído de campo.

\section{Resultados.}

1. Duración de los estadios de desarrollo de Amblyseius chungas.

La duración de huevo hasta la emergencia del adulto en hembras fue $6.60 \pm 0.57 \mathrm{~d}$ y en machos $6.46 \pm 0.44$ d (Tabla 1), estos valores no presentaron diferencias significativas $\left(F_{6,23}=0.172 ; \quad \mathrm{P}=0.982\right)$. Durante las pruebas realizadas se comprobó que la mayoría de larvas se alimenta de $P$. citri. Asimismo, en los subsecuentes estadios (protoninfa y deutoninfa) de ambos sexos se alimentan de $P$. citri.

Tabla 1. Duración (media \pm SEM) de los estadios inmaduros de Amblyseius chungas

\begin{tabular}{ccccccc}
\hline Estadios & $\mathrm{n}$ & $\begin{array}{c}\text { Hembra } \\
\text { Tiempo de } \\
\text { desarrollo } \\
\text { (días)* }\end{array}$ & $\mathrm{n}$ & $\begin{array}{c}\text { Macho } \\
\text { Tiempo de } \\
\text { desarrollo } \\
\text { (días)* }\end{array}$ & $\begin{array}{c}\text { F valores/ } \\
\text { Grados de } \\
\text { libertad }\end{array}$ & P \\
\hline Huevo & 66 & $2.38 \pm 0.19 \mathrm{a}$ & 61 & $1.80 \pm 0.15 \mathrm{a}$ & $\mathrm{F3,57}=0.711$ & 0.550 \\
Larva & 66 & $1.62 \pm 0.15 \mathrm{a}$ & 61 & $1.84 \pm 0.16 \mathrm{a}$ & $\mathrm{F} 2,58=0.652$ & 0.525 \\
Protoninfa & 49 & $1.62 \pm 0.22 \mathrm{a}$ & 54 & $1.50 \pm 0.17 \mathrm{a}$ & $\mathrm{F3,45}=0.593$ & 0.623 \\
Deutoninfa & 30 & $1.27 \pm 0.22 \mathrm{a}$ & 35 & $1.39 \pm 0.20 \mathrm{a}$ & $\mathrm{F} 3,26=2.488$ & 0.083 \\
Huevo-Adulto & 30 & $6.60 \pm 0.57 \mathrm{a}$ & 35 & $6.46 \pm 0.44 \mathrm{a}$ & $\mathrm{F} 3,57=0.711$ & 0.550 \\
\hline
\end{tabular}

* La letra a significa que los análisis de varianza entre hembra y macho del mismo estadio de desarrollo no mostraron diferencias significativas al nivel del 5\%. n: número de individuos por prueba.

2. Predación y reproducción de Amblyseius chungas. La respuesta funcional de las hembras adultas de $A$. chungas alimentadas con varias densidades de hembras adultas de $P$. citri (Tabla 2; Figura 2) mostró una curva que llega a un nivel constante (formando una meseta), y de acuerdo con el análisis de regresión logística y a la clasificación de Holling (1959, 1965) se determinó que correspondía a una respuesta funcional de tipo II (Tabla 3). El número de hembras adultas de $P$. citri consumidas por día se incrementó significativamente conforme se incrementó la densidad inicial de la presa $\left(F_{7,208}=79.686\right.$; $\left.\mathrm{P}<0.001\right)$. El consumo promedio del predador alcanzó una constante al alimentarse aproximadamente de 15 presas. De acuerdo con la regresión no lineal ajustada por cuadrados mínimos, usando como función general la ecuación citada anteriormente, los valores de $a \mathrm{y}$ Tm fueron $1.00 \pm 0.523$ /hora y $1.00 \pm 0.021$ horas, respectivamente. La respuesta reproductiva (huevos/predador/día) en este mismo experimento (Tabla 2; Figura 3), reflejó una respuesta tal como en la curva de respuesta funcional $\left(\mathrm{F}_{7},{ }_{206}=5.947\right.$; $\mathrm{P}<0.001$ ), sin embargo es fluctuante. Durante el experimento se observó que el umbral máximo de oviposición fue de 3 huevos/día.

La respuesta funcional de Amblyseius chungas a varias densidades de ninfas de $P$. citri (Tabla 2; Figura 4) mostró una curva que de acuerdo con el análisis de regresión logística y la clasificación de Holling (1959, 1965), se determinó que correspondía a una respuesta funcional de tipo II (Tabla 4). El número de ninfas de $P$. citri consumidas por día se incrementó significativamente conforme se fue incrementando la densidad inicial de la presa $\left(F_{5,157}=230.208\right.$; $\left.\mathrm{P}<0.001\right)$. El consumo promedio por predador alcanzó su mayor valor al alimentarse de 16 ninfas de la presa/día aproximadamente. De acuerdo con la regresión no lineal ajustada por cuadrados mínimos y usando como función general la ecuación citada anteriormente, los valores de $a$ y $T m$ fueron $1.00 \pm 0.594 /$ hora y $1.217 \pm$ 0.040 horas, respectivamente. La respuesta reproductiva en este experimento (Tabla 2; Figura 5) reflejó una tendencia semejante a la respuesta funcional. Cuando se compararon los valores de las diferentes pruebas de la respuesta reproductiva se observó diferencia significativa $\left(\mathrm{F}_{5,180}=13.817\right.$; $\mathrm{P}<0.001)$. Se observó que el umbral máximo de oviposición fue de 3 huevos/día, mientras que el promedio alcanzó 2 huevos/día.

De acuerdo con las observaciones de los experimentos (2.1) y (2.2) de respuesta funcional anteriormente mencionados, se puede observar la preferencia de $A$. chungas por estadios ninfales que por adultos de $P$. citri cuando son ofrecidas altas densidades de la presa (de 20 a 25 P. citri; Tabla 2), este hecho puede estar relacionado con el nivel de saciación del predador. Los valores de la tasa de búsqueda instantánea (a) y el tiempo de manipulación (Tm) de A. chungas fueron próximos tanto cuando se le alimenta con ninfas o adultos de $P$. citri.

El experimento (2.3) donde fue evaluada la predación y reproducción a diferentes densidades del predador $A$. chungas (Tablas 5-6, Figuras 6, 7), mostró que cuando el predador es alimentado de un número fijo de presas, la mayor cantidad de presas consumidas ocurre durante las primeras 24 horas. En los días subsiguientes se observó una menor tasa de predación, posiblemente debido a que las hembras quedaron saciadas $y / 0$ porque la hembra tuvo que emplear tiempo en la oviposición. El tiempo que tardó $A$. chungas en predar a los cincuenta $P$. citri fue disminuyendo conforme aumentaba la densidad de $A$. chungas. Este periodo varió significativamente de una 
prueba a otra $\left(F_{2,242}=28.533 ; \mathrm{P}<0.001\right)$, siendo la prueba de $\operatorname{diez} A$. chungas, la que menor tiempo registró, y la prueba que más tardó fue cuando se liberó sólo un $A$. chungas. La respuesta reproductiva en este experimento fue fluctuante, sin embargo, en el tratamiento de diez A. chungas se observó que a partir del segundo día de experimentación la oviposición aumentaba significativamente respecto al primer día. Por otro lado, se corroboró el canibalismo a partir del experimento con diez A. chungas, lo que conllevó a no continuar el análisis con mayor número de individuos de $A$. chungas. Probablemente el tamaño de la unidad experimental influyó en el comportamiento de canibalismo, debido a una competencia intraespecífica por espacio y alimento. acceso, como hojas enrolladas, debajo de cadáveres de $P$. citri, o de escamas de queresas Lepidosaphes beckii (Newman) (Hemiptera: Diaspididae). Durante la eclosión de la larva, ésta se auxilia con su primer par de patas. También se observó que la larva no se alimenta en las primeras horas, pero en las últimas horas generalmente se alimenta de huevos, larvas y ninfas de $P$. citri antes de mudar a protoninfa, tornando su cuerpo a un color rojo translúcido. Los huevos son ovalados casi transparentes en las primeras horas, tornándose blanquecinos pasadas algunas horas. Durante la crianza se observó que $A$. chungas se alimenta también de Polyphagotarsonemus latus (Banks) (Tarsonemidae), Eutetranychus banski (McGregor), Tetranychus spp. (Tetranychidae) y de

Tabla 2. Respuesta funcional y reproductiva de Amblyseius chungas a diferentes densidades de Panonychus $c$ (media \pm SEM).

\begin{tabular}{|c|c|c|c|c|c|c|c|c|}
\hline \multirow{2}{*}{$\begin{array}{l}\text { Densidades de } \\
P \text {. citri }\end{array}$} & \multicolumn{4}{|c|}{ A. chungas alimentado con $P$. citri adultos } & \multicolumn{4}{|c|}{ A. chungas alimentado con $P$. citri ninfas } \\
\hline & $\mathrm{n}$ & P. citri consumido/día* & $\mathrm{n}$ & Oviposición/día* & $\mathrm{n}$ & P. citri consumido/día* & $\mathrm{n}$ & Oviposición/día* \\
\hline 1 P. citri & 24 & $0.92 \pm 0.12 \mathrm{a}, \mathrm{A}$ & 24 & $0.13 \pm 0.14 a, A$ & 52 & $0.90 \pm 008 \mathrm{a}, \mathrm{A}$ & 52 & $0.08 \pm 0.07 \mathrm{a}, \mathrm{A}$ \\
\hline 5 P. citri & 25 & $3.92 \pm 0.38 \mathrm{ab}, \mathrm{A}$ & 25 & $0.56 \pm 0.29 a b, A$ & 25 & $4.56 \pm 0.36 \mathrm{~b}, \mathrm{~A}$ & 25 & $0.44 \pm 0.29 \mathrm{ab}, \mathrm{A}$ \\
\hline 10 P. citri & 25 & $5.92 \pm 0.67 \mathrm{bc}, \mathrm{A}$ & 25 & $0.64 \pm 0.29 a b, A$ & 23 & $7.43 \pm 0.92 \mathrm{c}, \mathrm{A}$ & 23 & $0.78 \pm 0.37$ abc, $\mathrm{A}$ \\
\hline 15 P. citri & 24 & $9.08 \pm 0.90 \mathrm{~cd}, \mathrm{~A}$ & 24 & $1.13 \pm 0.38 \mathrm{ab}, \mathrm{A}$ & 25 & $10.20 \pm 0.82 d, A$ & 25 & $1.04 \pm 0.37 b c, A$ \\
\hline 20 P. citri & 25 & $10.64 \pm 1.23$ de, $\mathrm{A}$ & 25 & $0.76 \pm 0.32 \mathrm{ab}, \mathrm{A}$ & 34 & $13.44 \pm 0.87 e, B$ & 33 & $1.00 \pm 0.34 b c, A$ \\
\hline 25 P. citri & 27 & $13.48 \pm 1.04 \mathrm{e}, \mathrm{A}$ & 27 & $0.93 \pm 0.33 \mathrm{ab}, \mathrm{A}$ & 26 & $15.58 \pm 0.97 e, B$ & 28 & $1.32 \pm 0.35 \mathrm{c}, \mathrm{A}$ \\
\hline 30 P. citri & 26 & $15.15 \pm 1.20 \mathrm{e}$ & 26 & $1.42 \pm 0.36 b$ & & & & \\
\hline 50 P. citri & 38 & $15.18 \pm 1.90 \mathrm{e}$ & 38 & $1.11 \pm 0.36 b$ & & & & \\
\hline
\end{tabular}

* Las letras a, b, c, d y e (leídas verticalmente) son los análisis de varianza de las pruebas de depredación con adultos o ninfas de $P$. citri. Las letras A y B en mayúscula (leídas horizontalmente) son los análisis de varianza de la predación y oviposición de A. chungas comiendo adultos y ninfas de $P$. citri, letras diferentes significa que hay diferencias significativas al nivel del 5\%. n: número de repeticiones por prueba.

Tabla 3. Regresión logística de la proporción de adultos hembras de citri consumidas por Amblyseius chunga

\begin{tabular}{lllcccc}
\hline Parámetro & g.l. & Estimador & ES & IC (95\%) & X2 & P \\
\hline $\begin{array}{l}\text { Intercepto } \\
\begin{array}{l}P \text {. citri } \\
\text { ofrecidos }\end{array}\end{array}$ & 1 & 1.0203 & 0.0740 & $0.8752,1.1654$ & 189.99 & $<0.0001$ \\
\hline
\end{tabular}

Tabla 4. Regresión logística de la proporción de ninfas de $P$. c consumidas por Amblyseius chungas

\begin{tabular}{llllclc}
\hline Parámetro & g.l. & Estimador & ES & IC (95\%) & X2 & P \\
\hline $\begin{array}{l}\text { Intercepto } \\
\begin{array}{l}\text { P. citri } \\
\text { ofrecidos }\end{array}\end{array}$ & 1 & 1.7984 & 0.1624 & $1.4801,2.1167$ & 122.63 & $<0.0001$ \\
\hline
\end{tabular}

\section{Comportamiento de Amblyseius chungas.}

El comportamiento de cópula en $A$. chungas es similar a la mayoría de fitoseídos. Los machos adultos se dirigen a la parte ventral de éstas y luego introducen sus quelíceros y a través de los espermidáctilos transfieren sus espermatóforos a las espermatecas de las hembras. De acuerdo a Amano \& Chant (1978) este comportamiento corresponde al tipo “Amblyseius-Typhlodromus”.

Cuando la hembra procede a ovipositar, deja generalmente los huevos aislados uno de otro, pero también se ha observado 2 a 5 huevos agrupados. La postura se realiza generalmente en zonas de difícil los primeros estadios de desarrollo de la "cochinilla harinosa” Planococcus citri (Risso) (Hemiptera: Pseudococcidae). Sin embargo no se ha corroborado que se alimente de ácaros de la familia Acaridae, ni Eriophyidae.

Discusión.

1. Duración de los estadios juveniles de Amblyseius chungas. El ciclo de huevo a adulto de Amblyseius chungas (6-7 días) constituyó virtualmente la mitad del tiempo que demora $P$. citri en alcanzar el estadio de adulto, que va de los 11 a los 15 días, según Salazar (1999). Este periodo más corto, representa probablemente una ventaja en el control biológico del ácaro plaga en estudio. A. chungas tiene un periodo de desarrollo de estadios juveniles aproximadamente equivalente a Neoseiulus cucumeris (Oudemans) alimentado con $P$. citri (Zhang et al., 2001), siendo este valor muy semejante a varias especies de fitoseídos usados en el control biológico de plagas (Tanigoshi, 1982). 
Tabla 5. Depredación y oviposición de diferentes densidades de Amblyseius chungas alimentadas con Panonychus citri.

\begin{tabular}{|c|c|c|c|c|c|c|c|}
\hline \multirow{3}{*}{$\begin{array}{c}\text { n } \\
\text { Día }\end{array}$} & \multirow{2}{*}{\multicolumn{2}{|c|}{$\begin{array}{c}1 \text { Amblyseius chungas } \\
19\end{array}$}} & \multirow{2}{*}{\multicolumn{2}{|c|}{$\begin{array}{l}5 \text { Amblyseius chungas } \\
26\end{array}$}} & \multirow{2}{*}{\multicolumn{3}{|c|}{$\begin{array}{l}10 \text { Amblyseius chungas } \\
14\end{array}$}} \\
\hline & & & & & & & \\
\hline & $\begin{array}{c}\text { P. citri } \\
\text { consumidas* }\end{array}$ & Oviposición* & $\begin{array}{c}\text { P. citri } \\
\text { consumidas* }\end{array}$ & Oviposición* & $\begin{array}{c}\text { P. citri } \\
\text { consumidas* }\end{array}$ & Oviposición* & Canibalismo * \\
\hline 1 & $19.58 \pm 1.68$ a & $0.47 \pm 0.25 \mathrm{a}$ & $32.19 \pm 3.12 \mathrm{a}$ & $3.16 \pm 0.87 \mathrm{a}$ & $40.50 \pm 2.99 a$ & $6.79 \pm 2.20 \mathrm{a}$ & $0.25 \pm 0.59 a$ \\
\hline 2 & $8.95 \pm 2.37 b$ & $1.47 \pm 0.61 \mathrm{ab}$ & $8.81 \pm 1.86 b$ & $4.23 \pm 1.09 \mathrm{a}$ & $7.36 \pm 2.25 b$ & $10.43 \pm 2.68 \mathrm{a}$ & $2.14 \pm 2.53 b$ \\
\hline 3 & $7.79 \pm 1.48$ b & $1.53 \pm 0.59 a b$ & $4.82 \pm 1.91 \mathrm{c}$ & $4.64 \pm 1.13 \mathrm{a}$ & $4.00 \pm 2.39 \mathrm{~b}$ & $9.29 \pm 5.62 \mathrm{a}$ & $4.00 \pm 1.30 \mathrm{c}$ \\
\hline 4 & $7.16 \pm 1.58 \mathrm{~b}$ & $1.32 \pm 0.58 \mathrm{ab}$ & $5.19 \pm 2.52 c$ & $4.81 \pm 2.16 a$ & & & \\
\hline 5 & $4.35 \pm 1.40 \mathrm{c}$ & $0.76 \pm 0.53 \mathrm{a}$ & $4.67 \pm 9.41 \mathrm{c}$ & $6.33 \pm 9.41 a$ & & & \\
\hline 6 & $2.83 \pm 1.04 \mathrm{c}$ & $0.17 \pm 0.25 \mathrm{a}$ & & & & & \\
\hline 7 & $2.00 \pm 2.20 \mathrm{c}$ & $0.50 \pm 0.88 \mathrm{a}$ & & & & & \\
\hline 8 & $1.50 \pm 2.05 \mathrm{c}$ & $1.00 \pm 2.25 \mathrm{a}$ & & & & & \\
\hline 9 & $1.00 \pm 0.00 \mathrm{c}$ & $1.50 \pm 6.35 \mathrm{a}$ & & & & & \\
\hline
\end{tabular}

* Las letras a, b y c son los análisis de varianzas entre las diferentes depredaciones y número de huevos puestos por $A$. chungas en los días de pruebas. Las letras diferentes leídas verticalmente indican promedios con diferencias significativas al nivel del 5\%. n: número de repeticiones por prueba.

A pesar que los ensayos fueron realizados bajo condiciones ambientales de alta humedad relativa, queda aún la interrogante sobre el desarrollo de $A$. chungas en condiciones de humedades relativas bajas, dado que $P$. citri se distribuye en varias regiones que reportan humedades relativas bajas en la costa peruana (Ej. La Irrigación Santa Rosa). Según algunos autores, las bajas humedades relativas pueden reducir las actividades de forrajeo (Mori \& Chant, 1966), fecundidad (Reuveny et al., 1996), e incluso longevidad (Boyne \& Hain, 1983) de diferentes especies de ácaros de la familia Phytoseiidae, siendo lo más afectado la viabilidad de los huevos (Moraes \& McMurtry, 1981). Según Guanilo \& Martínez (2007), A. chungas se distribuye preferentemente en áreas con humedades ambientales elevadas. Para estudios futuros sería recomendable determinar la sobrevivencia de esta especie considerando condiciones de baja humedad. Durante la experimentación se trabajó a humedades relativas semejantes a la que $A$. chungas se encuentra en el campo para observar el desarrollo de esta especie bajo condiciones favorables de desarrollo.

La observación que durante el estadio larval $A$. chungas se alimenta de $P$. citri, no es un patrón de todas las especies de ácaros Phytoseiidae. Schausberger \& Croft (1999) mencionaron que existen tres tipos de larvas de ácaros fitoseídos: (1) larvas que no se alimentan, (2) larvas que se alimentan facultativamente y (3) larvas que se alimentan obligatoriamente. A. chungas se le considera dentro del tipo (2); dado que la mayoría de las larvas observadas se alimentaron antes de mudar al estado de protoninfa, sin embargo hubieron algunos casos en que éstas no se alimentaron.

2. Clasificación de la respuesta funcional de Amblyseius chungas.
Las pruebas de respuesta funcional realizadas con $A$. chungas mostraron que la motivación de esta especie para atacar presas disminuye cuando las hembras se encuentran saciadas. Estos datos concuerdan con las observaciones realizadas por Fransz (1974) y Rabbinge (1976) para otras especies de ácaros predadores de la familia Phytoseiidae.

Estudios detallados de respuesta funcional con ácaros predadores han demostrado que la meseta formada depende del estadio de desarrollo, edad y sexo del ácaro predador (Blommers, 1976; Takafuji \& Chant, 1976; Eveleigh \& Chant, 1983). Pero existen otros factores que también pueden afectar, como la temperatura (Sabelis, 1985), la baja humedad que propicia a la pérdida de agua (Mori \& Chant, 1966) y la historia alimenticia del predador (Fransz, 1974).

La respuesta funcional tipo II descrita por Holling (1959; 1965) se caracteriza por tener otro parámetro además de la eficiencia de búsqueda. Se denomina "tiempo de manipulación" (Tm) y se define como el periodo dedicado a perseguir, dominar, consumir y digerir las presas, y prepararse para la siguiente búsqueda. Dado que el tiempo de búsqueda se reduce debido al tiempo manipulación, este tipo de respuesta origina un aumento desacelerado de las presas consumidas, hasta llegar a una asíntota en la cual se expresa la máxima tasa de ataque (T/Tm). Es así que tiempos de manipulación largos conducen a bajas tasas de ataque y viceversa. Esta relación genera una predación denso-dependiente inverso, lo que implica que, a altas densidades poblacionales, las presas tendrán una menor probabilidad de ser atacadas que cuando se encuentran en densidades bajas.

La respuesta funcional de tipo II observada en $A$. chungas, se explica cuando el ácaro predador consume aproximadamente todas las presas en un inicio, generando en la gráfica una creciente línea recta 
inicial que se flexiona y decae a medida que aumenta la densidad de presas. En el experimento con $P$. citri ninfas, la línea formó una notoria meseta. Este tipo de respuesta funcional es bastante común en ácaros de la familia Phytoseiidae. (Laing \& Osborn, 1974; Rabbinge, 1976; Croft \& Blyth, 1979; Friese \& Gilstrap, 1982; Badii \& McMurtry, 1988; Cédola \& Botto, 1996; Zhang et al., 1998; Cuéllar et al., 2002). A pesar de la gran cantidad de factores que puede influir en la respuesta funcional de un predador, el valor obtenido durante las pruebas, permite afirmar que $A$. chungas responde funcionalmente al incremento de densidades altas de $P$. citri. En un sentido práctico, estos modelos permiten estimar el número de hembras del predador a liberar con una frecuencia determinada y a cierta densidad de población de la plaga (Cuéllar et al., 2002).

De los resultados obtenidos en el presente trabajo se corroboró que la oviposición por hembra disminuyó cuanto mayor fue la densidad de los predadores en la unidad experimental. Además se observó canibalismo en las unidades experimentales, cuando se colocaron 10 hembras del predador. Este tipo de interferencia en la tasa de oviposición ha sido observada también por Kuchlein (1966) en hembras del ácaro fitoseído Galendromus occidentalis (Nesbitt).

Tabla 6. Periodo (media \pm SEM) de predación de 50 Panonychus citri por Amblyseius chungas

\begin{tabular}{|c|c|c|c|}
\hline & 1 Amblyseius chungas & 5 Amblyseius chungas & 10 Amblyseius chungas \\
\hline $\mathrm{N}$ & 19 & 26 & 14 \\
\hline $\begin{array}{c}\text { Periodo } \\
\text { (días) }\end{array}$ & $6.16 \pm 0.72 \mathrm{a}$ & $3.58 \pm 0.36 b$ & $2.50 \pm 0.30 \mathrm{c}$ \\
\hline
\end{tabular}

En este estudio, el potencial reproductivo de $A$. chungas tuvo valores equivalentes a los hallados en otras especies de ácaros generalistas del tipo III como Typhlodromus pyri (Scheuten) y Phytoseius hawaiiensis Prasad, sin embargo estos valores fueron menores valores a los observados en otras especies de fitoseídos como Iphiseius degenerans Berlese, Amblyseius largoensis (Muma), Amblydromalus limonicus (Garman \& McGregor) y Typhlodromalus peregrinus (Muma) (1.5 - 2 huevos/día) (McMurtry \& Rodríguez, 1987; Sabelis \& Janssen, 1994).

Se recomienda realizar pruebas de preferencia alimenticia a $A$. chungas, ofreciéndole especies de arañitas que producen abundante tela como es el caso de arañitas del género Tetranychus. Amblyseius chungas ha sido reportado en campo asociado con las siguientes especies fitófagas: Brevipalpus californicus (Banks), Czenspinskia sp., Eutetranychus banski (McGregor), P. citri, Polyphagotarsonemus latus (Banks), Tetranychus desertorum Banks y Tetranychus urticae Koch (Guanilo \& Martínez, 2007; Guanilo, 2008). Según con lo mencionado, en el caso de $A$. chungas no hay una marcada especificidad por el tipo de arañita roja al cual está asociado en su medio natural, dado que ha sido encontrado en asociación con arañitas rojas que producen gran cantidad de tela como $T$. desertorum, y T. urticae, así como con otras que no producen gran cantidad de tela como $P$. citri. McMurtry \& Croft (1997) indicaron que los ácaros predadores generalistas de tipo III pueden ser influenciados fuertemente por la anatomía de la hoja y que muchos de ellos suelen ser encontrados en el

3. Clasificación de los hábitos alimenticios de Amblyseius chungas.

Amblyseius chungas es un predador generalista cuyas características corresponden a un predador tipo III según McMurtry \& Croft (1997). Los ácaros predadores de tipo III se caracterizan por alimentarse de ácaros y pequeños insectos de diversas familias, lo que fue también observado en $A$. chungas, mediante las pruebas en este estudio y observaciones personales.

Al igual que otros predadores generalistas, en $A$. chungas también se observó canibalismo. Schausberger \& Croft (2000) estudiando tres especies generalistas (Amblyseius andersoni Chant, Neoseiulus cucumeris (Oudemanns), y Neoseiulus fallacis (Garman), determinaron que el canibalismo puede permitir una tasa de sobrevivencia equivalente a cuando se les alimenta con dietas adecuadas, sin embargo el tiempo de desarrollo se retarda, además, el canibalismo puede ser un mecanismo para estabilizar los niveles poblacionales y disminuir los riegos de extinción. envés de las hojas, en depresiones laterales de la nervadura media, entre tricomas y en áreas protegidas. Hecho verificado en $A$. chungas.

Amblyseius chungas ha sido reportado asociado en las siguientes plantas: Brugmansia arborea (L.), Capsicum annum L., Cestrum auriculatum L'Her., Citrus spp., Nicandra physalodes (L.), Ricinus communis L. y Solanum americanum Mill (Guanilo \& Martínez, 2007; Guanilo et al., 2008). Estos registros han demostrado que $A$. chungas habita plantas con hojas glabras y pilosas. Esta observación va en contradicción con la tendencia observada por McMurtry \& Croft (1997), quienes mencionaron que los fitoseídos generalistas tipo III habitan preferentemente plantas con hojas glabras como, árboles y arbustos cítricos, palto y café (Sabelis \& Bakker, 1992). conocimiento de los parámetros biológicos y de predación del ácaro predador $A$. chungas. Este ácaro predador ha sido únicamente encontrado en Perú, inclusive de acuerdo con trabajos anteriores fue el más
El estudio realizado es de relevancia para el 
abundante y frecuente en cítricos de la costa central peruana (Guanilo \& Martínez, 2007), donde $P$. citri es una plaga clave. Los estudios de predación de $A$. chungas son prueba fehaciente de su potencial como enemigo natural de $P$. citri, con mayor preferencia por los estadios inmaduros que por los adultos de la misma. Las observaciones dentro del presente estudio y los reportes encontrados muestran que este ácaro predador es generalista, lo que es un factor importante a ser considerado dentro de un programa de control biológico aplicado.

\section{Agradecimientos.}

A PROCITRUS, por su contribución económica y auspicio en la formulación de la presente investigación. A Blanca Cuadros, Carol Castillo, Luis Calle, Marivel Pereyra, Raquel Gonzáles y Teresa Rojas, que colaboraron en el mantenimiento de las crianzas de los ácaros en estudio. Al profesor Gilberto José de Moraes (Escola Superior de Agricultura "Luiz de Queiroz”, Universidade de São Paulo, Piracicaba, São Paulo, Brasil) y a la profesora Alicia Diestro Diestro (Universidad Nacional Mayor de San Marcos, Lima, Perú) por su valioso apoyo durante la ejecución del presente estudio.

\section{Literatura citada.}

Amano H. \& Chant D.A. 1978. Mating behavior and reproductive mechanisms of two species of predacious mites. Phytoseiulus persimilis Athias-Henriot and Amblyseius andersoni (Chant) (Acarina: Phytoseiidae). Acarologia. 20: 196-213.

Badii M.H. \& McMurtry J.A. 1988. Response of the predatory mite Phytoseiulus longipes Evans to spatial variation in the density of female prey Tetranychus pacificus McGregor (Acari: Phytoseiidae, Tetranychidae). International Journal of Acarology (West Bloomfield). 14: 57-60.

Blommers L. 1976. Capacities for increase and predation in Amblyseius bibens (Acarina: Phytoseiidae). Zeitschrift fuer Angewand te Entomologie (Berlin). 81: 225-244.

Boyne J.V. \& Hain F.P. 1983. Response of Neoseiulus fallacis (Acarina: Phytoseiidae) to different prey densities of Oligonychus ununguis (Acarina: Tetranychidae) and to different relative humidity regimes. Canadian Entomologist (Otawa). 115: 16071614.

Chávez P.N. 2003. Fluctuación poblacional de Panonychus citri McGregor (Acarina, Tetranychidae) "arañita roja "y Phyllocoptruta oleivora Ashmead (Acarina, Eriophyidae) "acaro del tostado" de acuerdo a la aplicación foliar de $\mathrm{Ca}, \mathrm{Mg}$ y micronutrientes en mandarina cultivar Clemenules, en el valle de ChancayHuaral. Tesis para optar el Grado Académico de Magister en Ciencias. UNALM.

Cédola C. \& Botto E. 1996. Respuesta funcional de Amblyseius idaeus y Phytoseiulus macropilis (Banks) [Acarina: Phytoseiidae] en condiciones de laboratorio. Revista Chilena de Entomología (Santiago). 23: 15-18.

Croft B.A. \& Blyth E.J. 1979. Aspects of the functional, ovipositional and starvation response of Amblyseius fallacis to prey density. In. Recent Advances in Acarology, Volume I. Edited by J. G. Rodriguez. Academic Press, New York. PP. 41-47.

Cuéllar M.E., Bellotti A.C. \& Melo E.L. 2002. Aspectos de la biología y el consumo de Neoseiulus cucumeris y Typhlodromalus aripo (Acari: Phytoseiidae) con la presa Thrips palmi (Thysanoptera: Thripidae). Revista Colombiana de Entomología. 28(1):1-7.

Denmark H.A. \& Muma M.H. 1989. A revision of the genus Amblyseius Berlese, 1914 (Acari: Phytoseiidae). Occasional Papers of the Florida State Collection of Arthropods, USA, 4.

Eveleigh E.S. \& Chant D.A. 1983. Experimental studies on acarine predatory-prey. interactions: distribution of search effort and depredation rates of a predator population in a patchy (Acarina: Phytoseiidae). A manual of Acarology, $2^{\text {nd }}$ ed. Oregon State University.

Fransz H.G. 1974. The functional response to prey density in an acarin system. Simulation Monographs, Pudoc, Wageningen.

Friese D.D. \& Gilstrap F.E. 1982. Influence of prey availability on reproduction and prey consumption of Phytoseiulus persimilis, Amblyseius californicus, and Metaseiulus occidentalis (Acari: Phytoseiidae). International Journal of Acarology (West Bloomfield). 8: 85-89.

Guanilo A.D. 2008. Busca de Tetranychus evansi e seus inimigos naturais no Peru e no norte da Argentina. Dissertação, Escola Superior de Agricultura "Luiz de Queiroz", Universidade de São Paulo, Piracicaba.

\& Martínez N. 2007. Predadores asociados a Panonychus citri McGregor (Acari: Tetranychidae) en la costa central del Perú. Ecología Aplicada (Lima). 6: $117-129$.

Moraes G.J. de \& Knapp M. 2008. Phytoseiid mites (Acari: Phytoseiidae) of the subfamily Amblyseiinae Muma from Peru, with descriptions of four new species. Zootaxa (Auckland). 1880: 1-47.

Holling C.S. 1959. Some characteristics of simple types of predation and parasitism. Canadian Entomologist (Ottawa). 91: 385-398.

1965. The functional response of predators to prey density and its role in mimicry and population regulation. Memoirs of the Entomological Society of Canada (Ottawa). 48: 3-60.

Kuchlein J.H. 1966. Mutual interference among the predacious mite Typhlodromus longipilus Nesbitt (Acari, Phytoseiidae). I. Effects of predator density on oviposition rate and migration tendency. Meded. Rijksfae. Landbouwwet. Gent. 31 (3): 740-746.

Juliano S.A. 1993. Nonlinear curve fitting: predation and functional response curves. En: Scheiner SM \& Gurevitch J (eds). Design and analysis of ecological experiments: 159-182. Chapman \& Hall, Nueva York.

Laing J.E. \& Osborn J.A.L. 1974. The effect of prey density on the functional and numerical responses of three species of predatory mites. Entomophaga (Paris). 19: 267-277.

McMurtry J. A. \& Croft B. 1997. Life styles of phytoseiid mites and their roles as biological control agents. Annual Review of Entomology (Palo Alto, California). 42: 291-321.

\& Rodriguez J. 1987. Nutritional ecology of phytoseiid mites. En: F Slansky (ed) Nutritional 
ecology of insects, mites and spiders. J Rodriguez, Wiley \& Sons, New York.

Moraes G.J. de \& McMurtry J.A. 1981. Biology of Amblyseius citrifolius (Denmark \& Muma) (Acarina: Phytoseiidae). Hilgardia (Berkeley). 49: 1-29.

Mori H. \& Chant D.A. 1966. The influence of humidity on the activity of Phytoseiulus persimilis Athias-Henriot and its prey, Tetranychus urticae Koch (Acarina: Phytoseiidae, Tetranychidae). Canadian Journal of Zoology (Toronto). 44: 863-871.

Morín Ch. 1985. Cultivo de Cítricos. 2da Edición. Instituto Interamericano de Cooperación para la Agricultura San José, Costa Rica. 566-567 pp.

ProCitrus. 2005. Investigación: Avances del proyecto de control biológico ProCitrus-SENASA. Citrinotas. Boletín informativo de ProCitrus (Lima). 27: 8.

Rabbinge R. 1976. Biological control of the fruit-tree red spider mite. Simulation Monographs, Pudoc, Wageningen.

Reuveny H., Palevsky E. \& Gerson U. 1996. Laboratory life history studies of the predaceous mite Typhlodromus athiasae (Acarina: Phytoseiidae). Systematic and Applied Acarology (Auckland). 1: 45-53.

Sabelis M.W. 1985. Predator-prey interaction: predation on spider mite. En: Helle W, Sabelis MW (eds), Spider Mites: Their Biology, Natural Enemies and Control. World Crop Pests, vol 1B: 103-129. Elsevier, Amsterdam.

\& Bakker F.M. 1992. How predatory mites cope with the web of their tetranychid prey: a functional view on dorsal chaetotaxy in the Phytoseiidae. Experimental and Applied. Acarology (Amsterdam). 16: 203-25.

\& Janssen A. 1994. Evolution of life history patterns in the Phytoseiidae. En Mites: Houck MA (ed) Ecological and evolutionary analyses of life-history patterns: 70-99. Chapman \& Hall, New York.
Salazar J.T. 1999. Control de las plagas de cítricos. Editorial Servicio Nacional de Sanidad Agraria - SENASA del Perú. Lima - Perú.

Schausberger P. \& Croft B.A. 1999. Activity, feeding, and development among larvae of specialist and generalist phytoseiid mite species (Acari: Phytoseiidae). Environmental Entomology (Lanham). 28 (2): 322329

2000. Cannibalism and intraguild predation among phytoseiid mites: are aggressiveness and prey preference related to diet specialization? Experimental and Applied Acarology (Amsterdam). 24: 706-725.

Solomon M.E. 1949. The natural control of animal populations. Journal of Animal Ecology (London). 18: $1-35$.

Takafuji A. \& Chant D.A. 1976. Comparative studies of two species of predacious phytoseiid mites (Acarina: Phytoseiidae), with special reference to their responses to the density of their prey. Research in Population Ecology (Sapporo, Japan). 17: 255-310.

Tanigoshi L.K. 1982. Advances in knowledge of the biology of the Phytoseiidae. En: Hoy MA (ed) Recent Advances in Knowledge of the Phytoseiidae: 1-22. Publication 3284, Univ. Calif. Press, Berkeley.

Zhang Y.-X., Zhang Z.-Q., Lin J.-Z. \& Liu Q.-Y. 1998. Predation of Amblyseius longispinosus (Acari: Phytoseiidae) on Aponychus corpuzae (Acari: Tetranychidae). Systematic and Applied Acarology (Auckland). 3: 53-58.

Chen C.-P, Lin J.-Z. \& Chen X. 2001. Amblyseius cucumeris (Acari: Phytoseiidae) as a biocontrol agent against Panonychus citri (Acari: Tetranychidae) on citrus in China. Systematic \& Applied Acarology (Auckland). 6: 35-44.

Figuras.

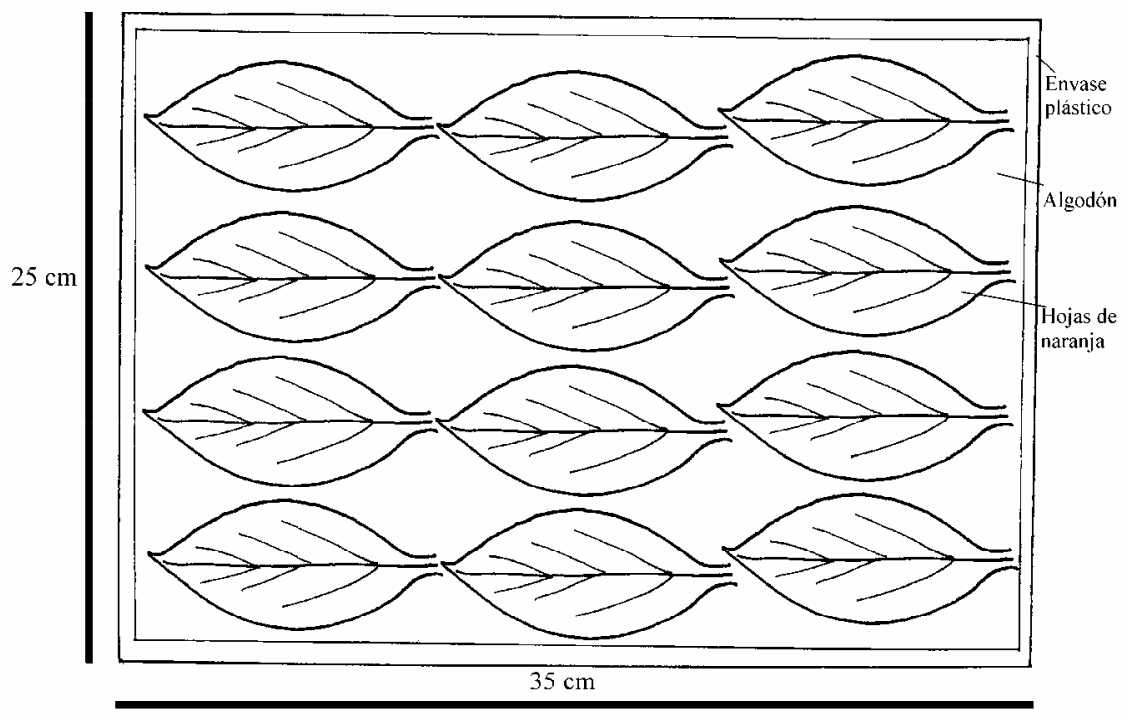

Figura 1. Metodología de crianza de Panonychus citri en laboratorio. 


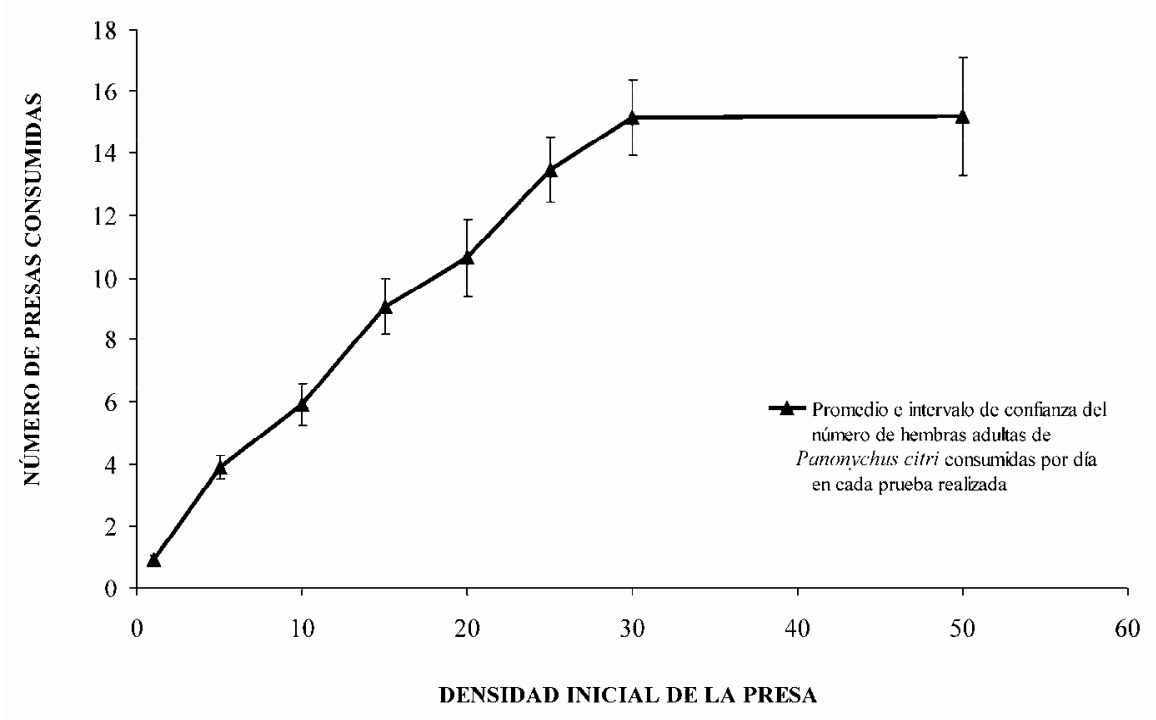

Figura 2. Respuesta funcional de Amblyseius chungas con adultos de Panonychus citri

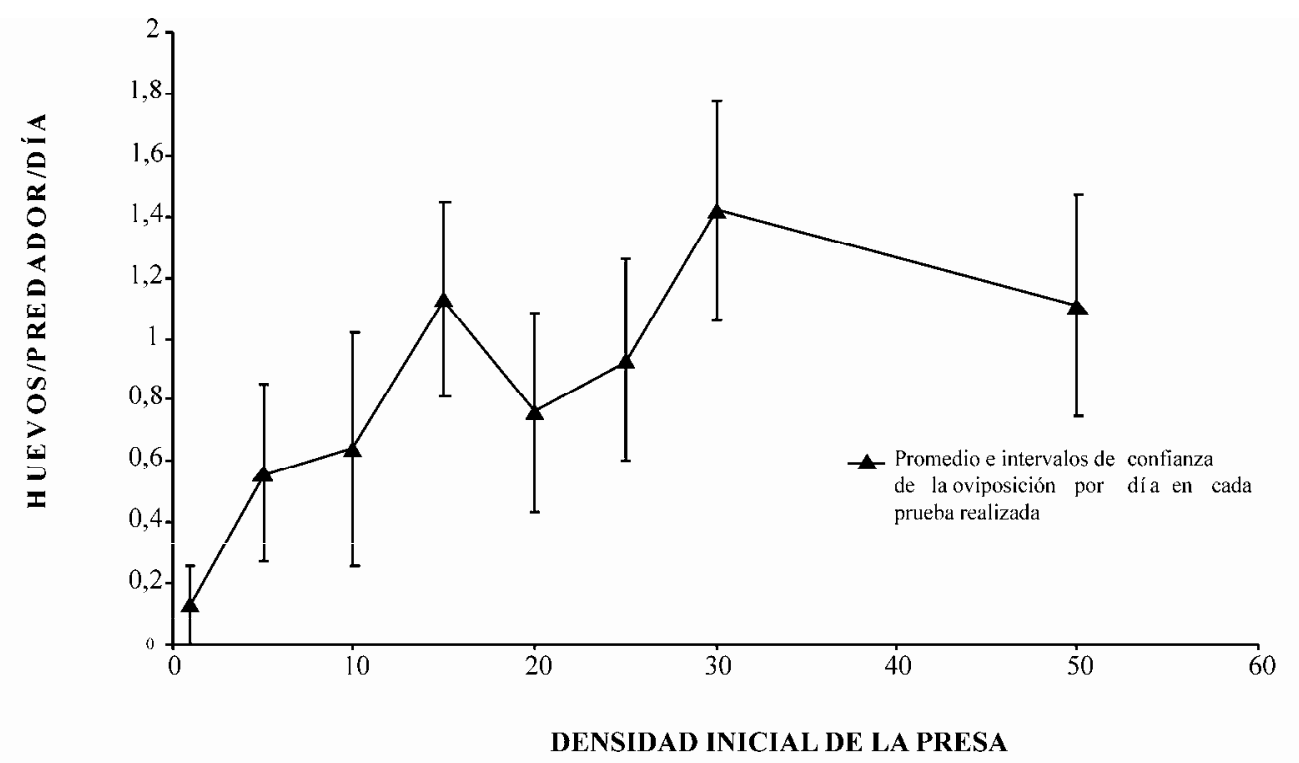

Figura 3. Oviposición de Amblyseius chungas con adultos de Panonychus citri. 




Figura 4. Respuesta funcional de Amblyseius chungas con ninfas de Panonychus citri

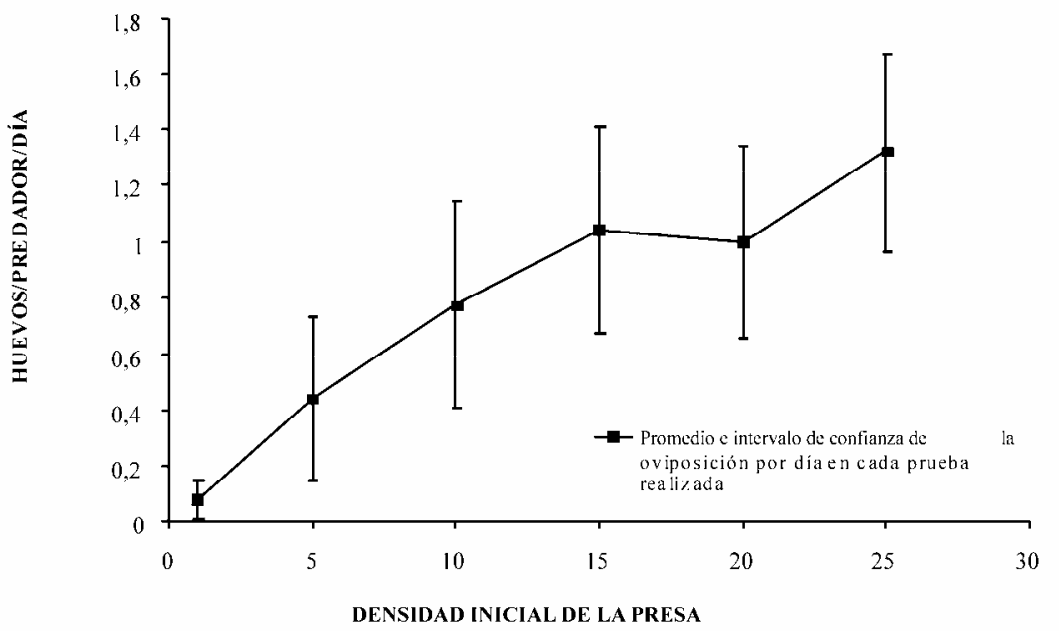

Figura 5. . Oviposición de Amblyseius chungas con ninfas de Panonychus citri. 


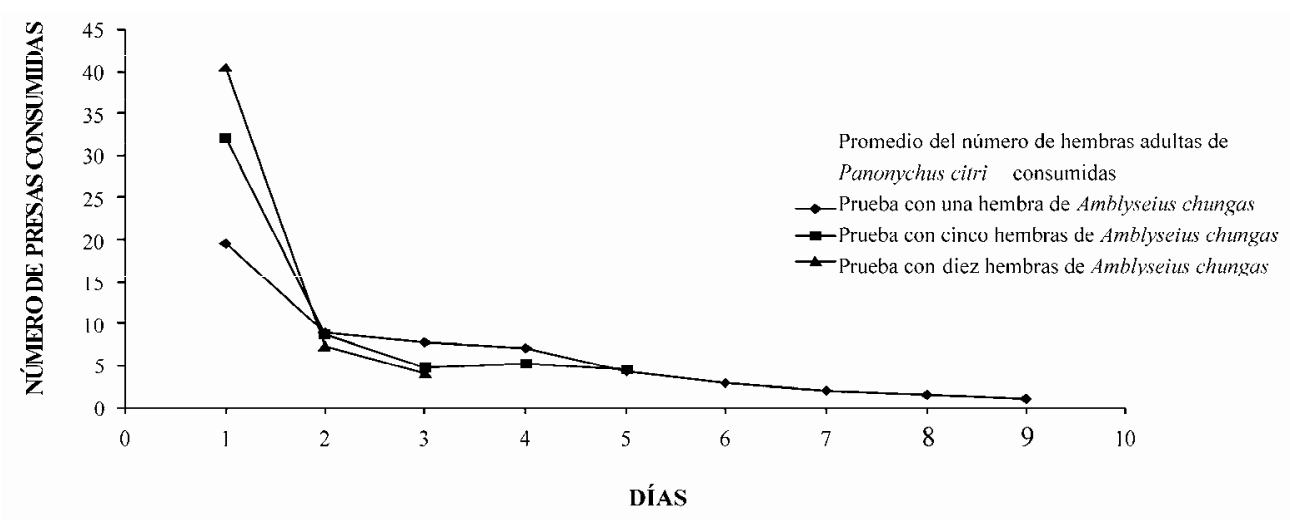

Figura 6. Periodo de predación de 50 adultos de Panonychus citri por Amblyseius chungas

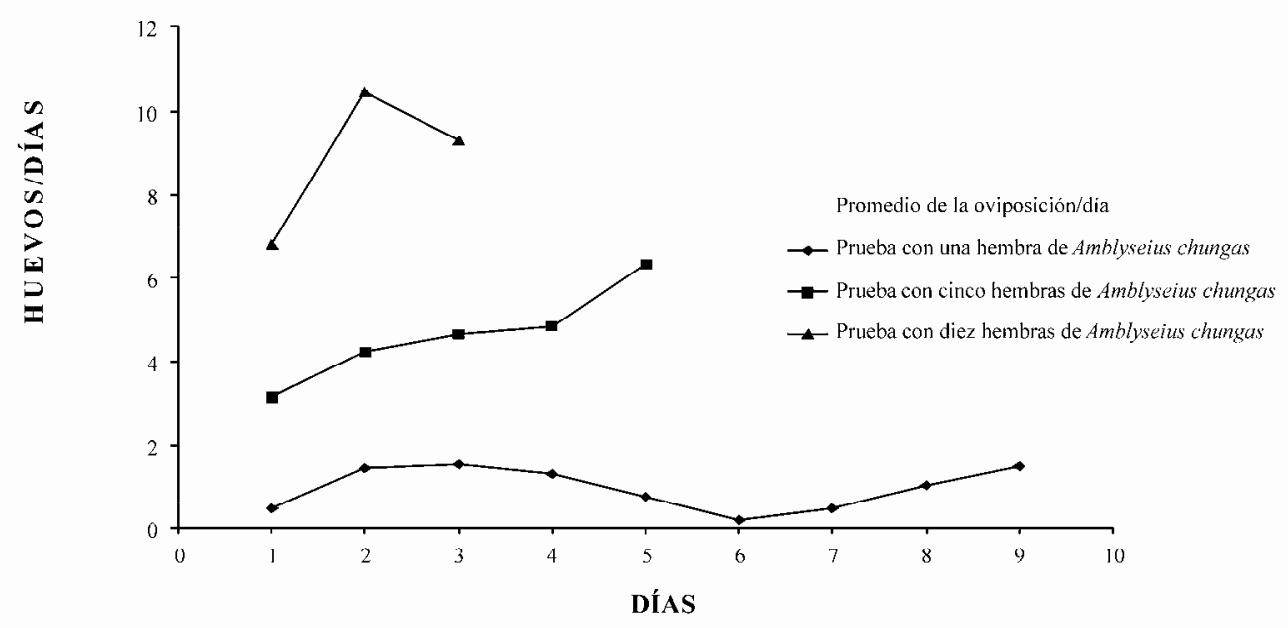

Figura 7. Oviposición de Amblyseius chungas alimentadas con 50 adultos de Panonychus citri.

\footnotetext{
${ }^{1}$ Bugs for Bugs Company - Bowen St Mundubbera, QLD 4626 Australia. a_guanilo@yahoo.co.uk.

${ }^{2}$ Facultad de Ciencias Biológicas, Universidad Nacional Mayor de San Marcos. nmartinezl@unmsm.edu.pe.
} 